\title{
A molecular scan in the Hubble Deep Field North
}

\author{
Roberto Decarli ${ }^{1}$, Fabian Walter ${ }^{1}$, Chris Carilli $^{2}$ and \\ Dominik Riechers ${ }^{3}$
}

\author{
${ }^{1}$ Max Planck Institut für Astronomie, Königstuhl 17, D-69117, Heidelberg, Germany \\ email: decarli@mpia.de \\ ${ }^{2}$ NRAO, Pete V. Domenici Array Science Center, P. O. Box O, Socorro, NM, 87801, USA \\ ${ }^{3}$ Cornell University, 220 Space Sciences Building, Ithaca, NY 14853, USA
}

\begin{abstract}
Our understanding of galaxy evolution has traditionally been driven by pre-selection of galaxies based on their broad-band continuum emission. This approach is potentially biased, in particular against gas-rich systems at high-redshift which may be dust-obscured. To overcome this limitation, we have recently concluded a blind CO survey at $3 \mathrm{~mm}$ in a region of the Hubble Deep Field North using the IRAM Plateau de Bure Interferometer. Our study resulted in 1) the discovery of the redshift of the bright SMG HDF850.1 ( $z=5.183)$; 2$)$ the discovery of a bright line identified as $\mathrm{CO}(2-1)$ arising from a BzK galaxy at $z=1.785$, and of other $6 \mathrm{CO}$ lines associated with various galaxies in the field; 3 ) the detection of a few lines (presumably $\mathrm{CO}(3-2)$ at $z \sim 2)$ with no optical/NIR/MIR counterparts. These observational results allowed us to expand the parameter space of galaxy properties probed so far in high- $z$ molecular gas studies. Most importantly, we could set first direct constraints on the cosmic evolution of the molecular gas content of the universe. The present study represents a first, fundamental step towards an unbiased census of molecular gas in 'normal' galaxies at high- $z$, a crucial goal of extragalactic astronomy in the ALMA era.
\end{abstract}

Keywords. galaxies: evolution - galaxies: ISM - ISM: evolution

\section{Introduction}

Most of our current understanding of the formation of galaxies is based on blank optical and near-IR deep observations of their stellar continua, and to less extent of their ionized gas and the dust continuum emission: The luminosities and colors of stellar continua are used to identify high- $z$ galaxies and to gauge their stellar contents and star formation rates (e.g., Steidel et al. 2004; Le Fèvre et al. 2005; Lilly et al. 2007). Narrow-band observations unveiled the presence of a population of star-forming galaxies with bright line emission arising from the ionized gas, but faint stellar continua (e.g., Ouchi et al. 2009, 2010). Wide field observations of the dust continuum gauge the obscured component of the star formation process (see review by Casey et al. 2014). The observational milestone resulting from all these investigations is the 'Lilly-Madau' plot, which traces the evolution of star formation rate (SFR) density in the universe as a function of cosmic time. These searches targeted star formation processes in act (through photoionized gas tracers or dust emission) or in the past (through stellar masses and ages). On the other hand, observations of the molecular gas, which is the fuel for star formation, have been limited to follow-up studies of galaxies that have been pre-selected from optical/NIR deep surveys, or to rare, extreme sources like bright sub-mm galaxies. Color-selection techniques (e.g., BzK, BMBX) have revealed significant samples of gas-rich, star forming galaxies at $z=1.5$ to $2.5\left(M_{\mathrm{H}_{2}}=10^{11} \mathrm{M}_{\odot}, M_{*}=10^{10} \mathrm{M}_{\odot}, \mathrm{SFR}=100 \mathrm{M}_{\odot} \mathrm{yr}^{-1}\right.$, Daddi et al. 2008, 2010a,b; Greve et al. 2010; Tacconi et al. 2010, 2013; Genzel et al. 2010, 2011, 2014; 
Decarli et al. 2014a). However, optical/NIR selections are likely missing gas-dominated and/or obscured galaxies, especially at intermediate to high redshift.

\section{A molecular scan in the HDF-N}

In order to overcome this limitation, we performed a volume-limited census of the molecular gas content in primeval galaxies, through a blind CO search over a large part of the $3 \mathrm{~mm}$ transparent window of the atmosphere $(79-115 \mathrm{GHz})$. The pointing center $(\mathrm{RA}=12: 36: 50.300, \mathrm{Dec}=+62: 12: 25.00$, J2000.0) was chosen to include the bright submm galaxy HDF850.1 (Hughes et al. 1998). The primary beam was $41^{\prime \prime}-60^{\prime \prime}$ in diameter. The typical beam size in our molecular line scan is $3^{\prime \prime} \times 2.7^{\prime \prime}(\sim 25 \mathrm{kpc}$ at $z=2$, and roughly constant at any $z \gtrsim 1$ ). The final cubes have a typical $\mathrm{rms}$ of $0.3 \mathrm{mJy}$ beam $^{-1}$ in $90 \mathrm{~km} \mathrm{~s}^{-1}$ channels, or $L_{\text {lim }}^{\prime}=(4-8) \times 10^{9} \mathrm{~K} \mathrm{~km} \mathrm{~s}^{-1} \mathrm{pc}^{2}$, assuming a typical line width of $300 \mathrm{~km} \mathrm{~s}^{-1}$, and by requiring a 3.5- $\sigma$ line detection (see Decarli et al. 2014b).

Our collapsed cube resulted in the deepest $3 \mathrm{~mm}$ continuum map available to date, with a $1-\sigma \mathrm{rms}$ of $8.5 \mu \mathrm{Jy}_{\text {beam }}{ }^{-1}$ (Decarli et al. 2014b). More importantly, we discovered various $\mathrm{CO}$ lines:

$i$ - We observed 2 lines arising from the bright sub-mm galaxy HDF850.1. We identified them as $\mathrm{CO}(5-4)$ and $\mathrm{CO}(6-5)$, which placed the source at $z=5.183$, as confirmed by follow-up observations of the $\mathrm{CO}(2-1)$ and [CII] lines (Walter et al. 2012; Neri et al. 2014). This was the first spectroscopic redshift measurement for this iconic source (14 years after its discovery by Hughes et al. 1998).

ii- We discovered 7 CO lines spatially associated with optical/NIR counterparts. One of these galaxies is a massive $\left(M_{*}=2.5 \times 10^{11} \mathrm{M}_{\odot}\right)$ star-forming $\left(38 \mathrm{M}_{\odot} \mathrm{yr}^{-1}\right) \mathrm{BzK}$ galaxy at $z=1.784$. A similarly bright $\mathrm{CO}$ line, on the other hand, is associated with a galaxy with $\sim 1$ order of magnitude smaller stellar mass at $z=2.044$ (Decarli et al. 2014b). This example shows that large molecular gas reservoirs can be found in galaxies with very different properties at $z \sim 2$ (Decarli et al. 2014b; Walter et al. 2014).

iii- Other line candidates were found in a spatial position which shows absolutely no optical/NIR/MIR counterparts, despite the exquisite depth of the available ancillary data. The most remarkable example, dubbed ID.18, has a flux of $0.58 \pm 0.11 \mathrm{Jy} \mathrm{km} \mathrm{s}^{-1}$ and is detected in independent data blocks. It peaks at $112.593 \mathrm{GHz}$. We argue that the line is $\mathrm{CO}(3-2)$ at $z=2.071$. A second transition, $\mathrm{CO}(4-3)$, is tentatively detected (at $\lesssim 4-\sigma$ level) at the same position in our $2 \mathrm{~mm}$ follow-up observations, thus confirming the line identification.

\section{CO luminosity functions and molecular gas content}

We compare our results with the empirical predictions by Sargent et al. (2014) and with semi-analytical cosmological models by Lagos et al. (2011) and Obreschkow et al. (2009a,b), as well as with the observed CO luminosity function at $z=0$ as measured by Keres et al. (2003). We place our constraints in the following way: We bin the CO blind detections in terms of CO luminosity, and we normalize to the volume of the universe in each redshift interval. For each luminosity bin, we place a lower limit corresponding to our secure detections (i.e., the lines that we managed to confirm via independent followup observations) and upper limits that represent the case where all line candidates are in fact real. Our blind detections probe a CO luminosity range close to the 'knee' of the predicted CO luminosity functions (Walter et al. 2014).

We convert the CO luminosity functions into a cosmic molecular content, $\rho_{\mathrm{H} 2}(z)$. This is the amount of molecular gas in galaxies per unit comoving volume. The luminosity 


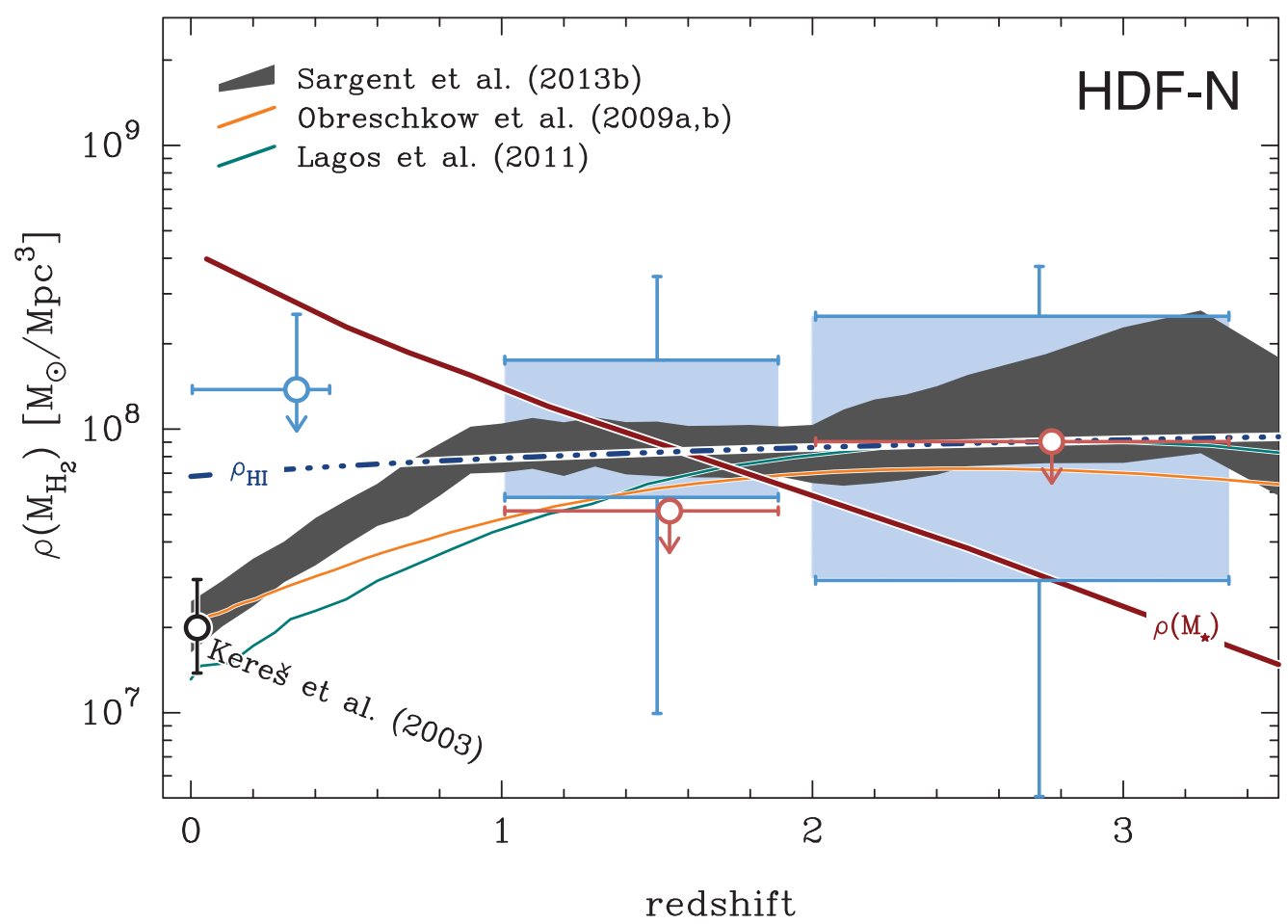

Figure 1. The evolution of the cosmic $\mathrm{H}_{2}$ mass density, $\rho_{\mathrm{H} 2}(\mathrm{z})$ based on predictions from semi-analytical comological models (Obreschkow et al. 2009a, 2009b, Lagos et al. 2011) as well as the empirical predictions by Sargent et al. (2014). The blue-shaded area shows only the contribution of our blind detections to $\rho\left(\mathrm{M}_{\mathrm{H} 2}\right)$, not corrected/extrapolated for a population of undetected sources at lower or higher $L_{\mathrm{CO}}^{\prime}$, while the limits from the CO stacks over a $H$-band flux-limited sample of galaxies are shown as red points and arrows. For a comparison, the evolution of the cosmic neutral gas mass density $\left(\rho_{\mathrm{HI}}\right)$ and of the stellar mass density $\left(\rho_{*}\right)$ are also plotted. Figure taken from Walter et al. (2014).

functions from empirical predictions or semi-analytical models are converted into $\mathrm{H}_{2}$ mass via standard Galactic correction factors, and then integrated over the whole $L_{\mathrm{CO}}$ range. On the other side, we do not attempt to correct for sources not detected in our scan at both lower and higher $L_{\mathrm{CO}}^{\prime}$ luminosities, given the unknown shape of the luminosity function. A comparison to empirical predictions of $\rho_{\mathrm{H} 2}(z)$ shows that the securely detected sources in our molecular line scan already provide significant contributions to the predicted $\rho_{\mathrm{H} 2}(z)$ in the redshift bins $\langle z\rangle \approx 1.5$ and $\langle z\rangle \approx 2.7$. Accounting for galaxies with CO luminosities that are not probed by our observations results in cosmic molecular gas densities $\rho_{\mathrm{H} 2}(z)$ that are higher than current predictions (see Fig. 1 and Walter et al. 2014). We note however that the current uncertainties (in particular the luminosity limits, number of detections, as well as cosmic volume probed) are significant.

\section{Conclusions}

Our molecular line scan of the HDF-N allows us to place first direct limits on CO luminosity function and on the molecular gas density in 'normal' galaxies at high redshift without any pre-selection based on optical/NIR/MIR wavelength observations. Our data shows that galaxies close to the peak of star formation activity $(z=1-3)$ have much higher molecular gas content than galaxies at $z=0$. This evolution matches or even 
exceeds the one predicted by our current understanding of galaxy properties. We note however that the current uncertainties in our precursor study are significant, and that current models can thus not be ruled out given the available data. The emerging capabilities of the Jansky Very Large Array (JVLA) and of the Atacama Large (Sub-)Millimeter Array (ALMA) will enable similar molecular deep field studies to much deeper levels and larger areas (e.g., da Cunha et al. 2013).

\section{Acknowledgements}

This IAU proceeding is based on the results presented in Decarli et al. (2014b) and Walter et al. (2014). We thank our collaborators M. Aravena, E. Bell, F. Bertoldi, D. Colombo, P. Cox, E. da Cunha, E. Daddi, D. Downes, M. Dickinson, R. Ellis, L. Lentati, R. Maiolino, K. M. Menten, R. Neri, H.-W. Rix, M. Sargent, D. Stark, B. Weiner, and A. Weiß. This study is based on observations with the IRAM Plateau de Bure Interferometer (PdBI). IRAM is supported by INSU/CNRS (France), MPG (Germany) and IGN (Spain). Support for RD was provided by the DFG priority program 1573 'The physics of the interstellar medium'.

\section{References}

Carilli, C. \& Walter, F. 2013, ARA\&A, 51, 105

Casey, C., et al. 2014, PhR, 541, 45

Daddi, E., et al. 2008, ApJ, 673, L21

Daddi, E., et al. 2010a, ApJ, 713, 686

Daddi, E., et al. 2010b, ApJ, 714, L118

da Cunha, E., et al. 2013, ApJ, 765, 9

Decarli, R., et al. 2014a, ApJ, 780, 115

Decarli, R., et al. 2014b, ApJ, 782, 79

Genzel, R., et al. 2010, MNRAS, 407, 2091

Genzel, R., et al. 2011, ApJ, 733, 101

Genzel, R., et al. 2014, arXiv:1409.1171

Greve, T., et al. 2010, ApJ, 719, 483

Hughes, D. H., et al. 1998, Nature, 394, 241

Keres, D., Yun, M. S., \& Young, J. S. 2003, ApJ, 582, 659

Lagos, C. D. P., et al. 2011, MNRAS, 418, 1649

Le Feèvre, O., et al. 2005, Nature, 437, 519

Lilly, S. J., et al. 2007, ApJ Suppl., 172, 70

Neri, R., et al. 2014, A\&A A, 562, 35

Obreschkow, D., et al. 2009a, ApJ, 702, 1321

Obreschkow, D., et al. 2009b, ApJ, 698, 1467

Ouchi, M., et al. 2009, ApJ, 706, 1136

Ouchi, M., et al. 2010, ApJ, 723, 869

Sargent, M. T., et al. 2014, ApJ, 793, 19

Steidel, C. C., et al. 2004, ApJ, 604, 534

Tacconi, L., et al. 2010, Nature, 463, 781

Tacconi, L., et al. 2013, ApJ, 768, 74

Walter, F., et al. 2012, Nature, 486, 233

Walter, F., et al. 2014, ApJ, 782, 79 